\title{
The Relationship between Stress and Diabetes Mellitus
}

\begin{abstract}
Keywords: Stress; Diabetes mellitus; Metabolic syndrome; Cortisol; HPA axis; Autoimmunity

\section{Summary}

Diabetes mellitus (DM) is a metabolic disease characterized by chronic hyperglycemia that results from an alteration of the secretion or action of insulin. This metabolic condition is not homogeneous and the World Health Organization distinguishes two main types of diabetes: Type 1 and Type 2 DM (TIDM and T2DM). Despite etiological and clinical differences, these two diseases share some characteristics including the role played by stress in the occurrence of the disease, its progression and chronicity, which involves habit changes and influences psychological and social life. In this review we will investigate the psychologic al correlates of TIDM and T2DM. We will focus on the role of stress in the disease and the need forglobal care plans in diabetic patients in order to improve their quality of life and metabolic control.
\end{abstract}

\section{Introduction}

Diabetes mellitus (DM) is a metabolic disease characterized by an inability to maintain normal glucose homeostasis that results from an alteration of the secretion or action of insulin, the hormone responsible for the uptake of glucose in the body [1].

This metabolic condition is not homogeneous and the World Health Organization distinguishes two main types [2] of diabetes. Type $1 \mathrm{DM}(\mathrm{T} 1 \mathrm{DM})$ is an autoimmune disease that occurs mostly in childhood or youth [3] and is characterized by the cell-mediated destruction of insulin-producing $\beta$-cells, leading to impaired glucose homeostasis, insulin insufficiency, and other complications [4]. T1DM sometimes also clusters with other autoimmune disorders [5]. The consequences of the impaired assimilation of sugar are: paradoxical polyphagia, polyuria, polydipsia, and ketosis. Once established, the disease is irreversible and it will require lifelong insulin replacement therapy by injection [6]. Type 2 DM (T2DM), in contrast, usually manifests itself in adults [7]. It does not involve autoimmune destruction of $\beta$-cells but it is due to a combination of both insulin resistance and an inability of the $\beta$-cells to compensate adequately with increased insulin release. It is related to family predisposition, sedentary lifestyle and obesity [8]. Insulin secretion is not completely compromised, but just altered. Sometimes, the pancreas does not produce adequate amounts in relation to the carbohydrates ingested. Other times, the release of insulin is normal, but the body becomes resistant and does not react properly. Generally, T2DM can be controlled through a balanced diet, weight reduction and drugs which stimulate hormone production or reduce insulin resistance. Insulin therapy is necessary only in a few cases, in particular for long term disease, when a secondary pancreatic failure occurs.

Epidemiological studies indicate an increased incidence of both types of DM though T2DM shows a greater incidence and prevalence, particularly in western countries [7]. Modern lifestyle including unhealthy diet, a sedentary lifestyle and stress contribute

\section{Journal of}

Neurology and Psychology

\section{Gemma Falco², Piero Stanley Pirro ${ }^{3}$, Elena Castellano $^{1}$, Maura Anfossi ${ }^{2}$, Giorgio Borretta ${ }^{1}$ and Laura Gianotti*}

${ }^{1}$ Division of Endocrinology, Diabetology and Metabolism, Department of Internal Medicine, S.Croce \& Carle Hospital, Cuneo, Italy

${ }^{2}$ Department of Medical Psychology, Directorate of Medical Services, S.Croce \& Carle Hospital, Cuneo, Italy

${ }^{3}$ Consultant for the Division of Endocrinology, Diabetology and Metabolism, Department of Internal Medicine, S.Croce \& Carle Hospital, Cuneo, Italy

\section{*Address for Correspondence}

Laura Gianotti MD, Division of Endocrinology, Diabetology and Metabolism, Department of Internal Medicine, S.Croce \& Carle Hospital Via Coppino 26, 12100 Cuneo, Italy, E-mail: laura.gianotti@unito.it

Copyright: (c) 2015 Falco G, et al. This is an open access article distributed under the Creative Commons Attribution License, which permits unrestricted use, distribution, and reproduction in any medium, provided the original work is properly cited.

\section{Submission: 09 February 2015}

Accepted: 14 April 2015

Published: 18 April 2015

Reviewed \& Approved by: Dr. Alicia K Smith, Assistant Professor of Psychiatry and Behavioral Sciences, Emory University, USA

to T2DM occurrence and development [8]. Despite etiological, clinical and epidemiological differences, T1DM and T2DM share some characteristics, including the role played by stress during their onset and progression, chronicity, which involves habit changes and influences psychological and social life and the presence of similar complications, which increase the risk of co-morbidity and mortality.

In this review, we will investigate the psychological correlates of T1DM and T2DM. We will concentrate on the role of stress and on the need for global care plans in diabetic subjects in order to improve their quality of life and metabolic control.

\section{The Concept of Stress}

The Canadian physiologist Hans Selye was the first scientist to study the effects of psychological stress on the human body in 1936 [9]. He made a distinction between stress, stressor and stress reaction which he considered as a complex phenomenon consisting of a set of nonspecific responses that result when the subject faces the situation. In front of a danger, the system enters into a defense condition, trying to restore balance in different ways. It is an adaptive mechanism, in which a series of physical changes predisposes the body to a "fight or flight" reaction. However, if the state of arousal continues over time, it can lead to negative consequences which Selye defines "General Adaptation Syndrome".

The author distinguishes three phases in response to a stressor. The first is an "alarm reaction": the homeostatic balance is altered, generating a physiological mobilization comparable to a "natural biological doping". Several functions (blood glucose, blood pressure, muscle mobility, heartbeat, etc.) increase the availability of energy, making it easier to face the situation. If the danger disappears, homeostasis is restored and the body returns to normal. On the 
contrary, if the stressful stimulus persists, we enter the second stage, called "resistance or adaptation" in which the pattern of biological reactions is modified. As long as the danger is present, visceral functions and physico-chemical parameters are maintained in an altered condition. This implies a considerable energy expenditure and a higher central and peripheral functional level. However, the individual cannot cope with the threatening environment forever and his resilience varies according to genetic, cognitive and psychosocial factors. In the long run, it results in an "exhaustion" of adaptability (third phase), and the subject can contract illness or even die. This happens especially when the source of the threat is inevitable, unwanted and repetitive [10], while, if the stress is short-lived, the body returns back to normal without any negative consequences.

In recent years, researchers have confirmed Selyes' hypothesis, showing more and more clearly that chronic stress may favor the onset of somatic disorders in susceptible subjects $[11,12]$. Prolonged exposure to adverse events, in fact, affects hormonal balance, metabolism and immunefunction [13]. The prolonged activation of the hypothalamus-pituitary-adrenal (HPA) axis, in particular, increases glucocorticoid levels, causing pathologies related to hypercortisolism. Furthermore, this condition promotes the alteration of the immune function and facilitates the development of central obesity, peripheral tissue resistance to insulin and glucose intolerance. These processes, however, are not the same in all individuals and researchers have found some gender-related variations [14]. Some authors actually believe that gender differences in the stress system may explain, at least in part, the greater vulnerability of men to vascular and infectious disease and the greater susceptibility of women to autoimmune diseases [15]. In fact, emerging data from molecular studies show that estrogenic hormone plays a central role in the development of autoimmune disease [16].

A brief overview on the functioning of the endocrine system and physiological mechanisms involved in the stress reaction follows.

\section{The endocrine system}

The word endocrine derives from the Greek words "endo," meaning "within", and "crinis," meaning "to secrete".

The endocrine system plays a role in regulating mood, growth and development, tissue function, metabolism, sexual and reproductive processes [17].

The foundations of the endocrine system are the hormones and glands. As the body's chemical messengers, hormones transfer information and instructions from one set of cells to another. Many different hormones move through the bloodstream, but each type of hormone is designed to affect only certain cells.

Some types of glands release their secretions in specific areas. For instance, exocrine glands, such as the sweat and salivary glands, release secretions in the skin or inside the mouth. Endocrine glands, on the other hand, release hormones directly into the bloodstream where they can be transported to cells in other parts of the body.

Roughly simplifying, the endocrine system is made up of the pituitary gland, the thyroid gland, the parathyroid glands, the adrenal glands, the pancreas, the ovaries (in females) and the testicles (in males), the adypocites and the hypothalamus. The latter is a collection of specialized neurones located in the lower central part of the brain and it is the main link between the endocrine and the nervous systems. Nerve cells in the hypothalamus control the pituitary gland by producing chemicals that either stimulate or suppress hormone secretions by the pituitary.

The pituitary makes hormones that control several other endocrine glands. Their secretion can be influenced by several factors, including psychological and physical stimuli. The pituitary is divided into two parts: the anterior lobe and the posterior lobe. The first one regulates the activity of the thyroid, the adrenals, and the reproductive glands. The second one, instead, releases antidiuretic hormone and oxytocin.

A negative feedback regulates the amounts of hormones available by detecting when blood levels rise above a threshold and inhibiting hormone production. This prevents hormone levels in the blood from continuing to rise, which could result in illness. Likewise, a positive feedback may occur [17].

\section{The physiology of stress}

In a physiological perspective, stress reaction is organized into two branches: the one governed by the sympathetic nervous system, that operates quickly, and the one governed by neuroendocrine axis (HPA), that activates a delayed response.

The first one starts in the parvocellular nucleus of the hypothalamus, which is connected by a bundle of nerve fibers to the locus coeruleus, in the spinal cord. From here, the adrenal medulla is stimulated, so that it produces catecholamines (adrenalin, noradrenalin, and dopamine), with physiological arousal (Figure 1).

The functioning of the HPA axis, instead, begins from the paraventricular nucleus of hypothalamus, which releases corticotropin-releasing hormone $(\mathrm{CRH})$ and arginine vasopressin (AVP). These substances stimulate the pituitary gland to produce adrenocorticotropic hormone (ACTH), that is released into the bloodstream and induces the adrenal cortex to secrete cortisol $[17,18]$.

The system is integrated because hypothalamic $\mathrm{CRH}$ and norepinephrine stimulate each other, according to a positive feedback mechanism. However, there is also a negative feedback, which prevents the physiological activation from lasting too long and damaging the body.

The hypothalamus, in fact, has particular receptors which detect cortisol levels and, depending on the case, activate the axis more or deactivate it altogether.

\section{Stress and the Onset of DM}

\section{Type 1 Diabetes Mellitus. The relationship between stress and autoimmunity}

The functioning of the immune system is extremely complex and it consists of several circuits, which are activated according to the noxious stimulus. For example, when faced with a virus or an intracellular parasite, the body prepares an inflammatory response, regulated by TH1 lymphocytes. If, instead, the threat consists of a bacterium or an extracellular parasite, an antibody response is activated, mediated by TH2 lymphocytes. Between these two systems, there is a balance, ensured by a mutual inhibition [18]. 


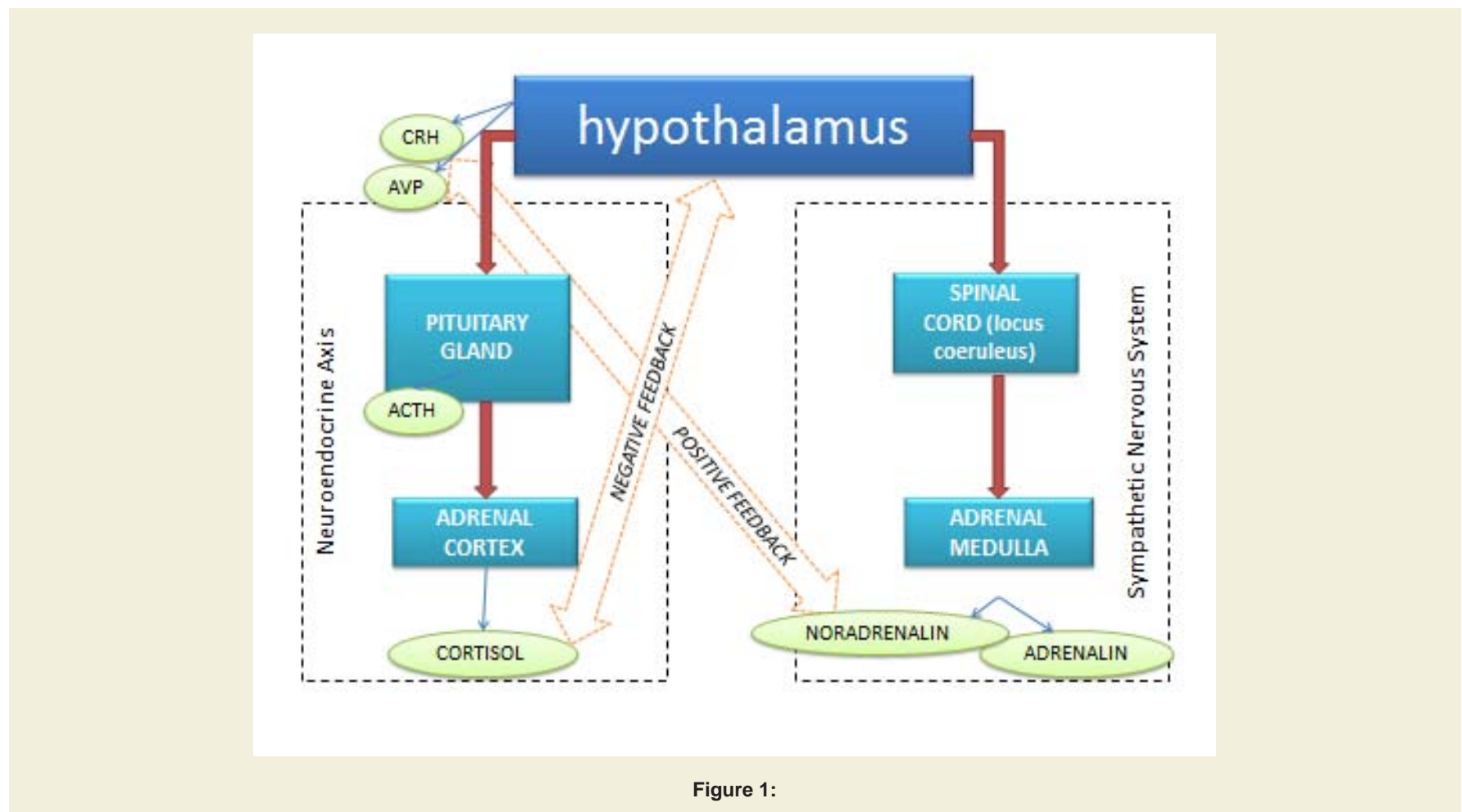

It has been shown that the activation of the HPA axis during stress reaction can change the homeostasis between the two systems and alters the activity of suppressive cells (T-suppressors) that usually prevent damage to the individual. Accordingly, epidemiological studies indicate that severe stress often precedes the development of certain Th1-mediated autoimmune diseases [19-21]. Among these, T1DM is characterized by the development of antibodies against islet cells in the pancreas and autoantibodies against insulin. However, the link between stress and T1DM (and, more generally, between stress and autoimmunity) cannot be reduced down to a linear causality. In fact, it depends on many variables related to the stressor, the physical and mental characteristics of the patient, as well as to his resilience and to the presence of a supportive family environment. Moreover, it is not certain that psychological events implicated in the development of the disease immediately precede the onset. On the contrary, these events may take place throughout the patient's entire life and, in particular, in childhood [22]. Psychological stress in children, in fact, can affect their immune functions, altering the activity of the antigens GAD65, HSP60 and IA-2, responsible for diabetes-related autoimmunity [23]. Furthermore, early negative experiences result in lifelong changes in coping strategies. Many studies show that people who have suffered stressful situations during childhood have a greater psychological vulnerability, combined with hyper-vigilance in the face of danger and low propensity to seek help [24]. These elements, in turn, facilitate the development of the physical disease or otherwise have an impact on the emotions arising out of it. It is to be remembered, however, that there is still controversy within the scientific community about the relationship between stress and T1DM [25].

\section{Type 2 Diabetes Mellitus: direct and indirect impact of stress}

T2DM is characterized by a chronic hyperglycemia, insulin resistance and a relative insulin secretion defect. At present, the causes of T2DM are not entirely clear, but predictors have been found in recent studies. Among these, obesity, hypertension, sedentary lifestyles, alterations in the glycemic status and lipid metabolism correlate with T2DM and its diffusion.

Insulin resistance and other conditions with minor degrees of glucose intolerance commonly occur together with a collection of clinical and biochemical features, that have been called Metabolic Syndrome (MS). This term defines a cluster of components that reflect overnutrition, sedentary lifestyles and an excess of adiposity [26]. MS affects about a quarter of the world's adult population, with significant variations according to age, gender and body mass index [27]. The prevalence of the MS is growing to epidemic proportions all over the world, both in the urbanized than in developing nations. MS predisposes to T2DM with concomitant cardiovascular diseases (CVD) and generates a cluster of cardiovascular risk factors whose core components are impaired glucose metabolism, obesity, dyslipidemia, and hypertension. MS is also associated with other co-morbidities, such as a prothrombotic and proinflammatory state, nonalcoholic fatty liver disease and other disorders in the renal, visual and reproductive systems.

A clearly defined pathophysiology and universal definition of MS is still lacking. As a result, several definitions for MS have been proposed by various international regulatory bodies: the National Cholesterol Education Program Adult Treatment Panel III (NCEP ATP III) describes Metabolic Syndrome as the presence of any three of the following components: abdominal obesity, dyslipidemia (high 
levels of triglycerides, low HDL), hypertension, and elevated fasting glucose [28]; the International Diabetes Federation (IDF) considers central obesity a mandatory component for the diagnosis of MS along with any two of the other components: hypertension, abnormal blood glucose, high serum triglycerides and low high density lipoprotein cholesterol [29]; recently, the IDF, the National Heart, Lung and Blood Institute (NHLBI), the American Heart Association (AHA), the World Heart Federation (WHF), the International Atherosclerosis Society (IAS) and the International Association for the Study of Obesity (IASO) have proposed a new harmonized definition which requires any three of the five components included in the IDF definition for the diagnosis of MS and don't consider central obesity an obligatory component [30].

As previously said, MS is usually a pre-clinical condition. Both genetic predisposition and lifestyle (such as being overweight, living a sedentary lifestyle and adopting bad dietary habits among others) lead individuals to develop T2DM.

Stress is one of the triggers [31] and it has been linked to a higher risk of T2DM, especially in women [32,33]. Nervous strain seems to have a direct and indirect influence on the probability of becoming ill. The reaction to a stressor may consist, in some cases, in the development of an unhealthy lifestyle, including the neglect of physical well-being, and eating in a disorderly fashion, often using food in a compensatory or consoling manner. These factors indirectly affect the risk of developing the disease [34]. In addition, physiological changes triggered by stress may directly affect the endocrine and immune systems [35].

Cortisol is one of the main actors mediating the effect of stress on metabolism in general, and on glucose metabolism in particular. Cortisol raises blood glucose levels by stimulating hepatic gluconeogenesis, and inhibiting the action of insulin [36]. These reactions - useful for initiating a fight or flight reaction - are not entirely suited to cope with the stressors triggered by modern life, which are mostly relational, intangible and durable. The pressing rhythms imposed by sedentary work, for example, do not involve an increase in energy requirements. The glucose mobilized from the liver is not used and remains in the bloodstream, causing a rise in blood sugar. Moreover, the way by which individuals evaluate events may influence these reactions: an anxious person may anticipate difficulties and amplify the feeling of danger in the face of everyday situations. This anxiety may generate a state of perpetual alarm, that can induce chronic hypercortisolism, likely to facilitate the onset of metabolic syndrome and T2DM.

\section{The Disease as a Source of Stress}

From a biopsychosocial perspective, it is important to consider, on the one and, that stress is the component that may trigger T1DM and T2DM, but, on the other hand, that it can also be the result of the disease itself. Getting ill, in fact, may cause personal and interpersonal conflicts, where the normal rhythms of life and habits are disrupted, forcing the individual to question personal values and long-term objectives [12].

T1DM and T2DM require a complex and largely self-managed treatment which includes the daily use of drugs (insulin or hypoglycemic agent), the regular measurement of blood glucose levels through invasive means and special attention to diet and everyday activities.

In Type 2 diabetics - that usually affects elderly people - changing established routines may create emotional and cognitive fatigue. In fact, they should reduce the intake of carbohydrates and learn new dietary guidelines and new procedures for the self-administration of drugs. On the other hand, Type 1 diabetics have no dietary restrictions, but they must make sure that insulin units are proportional to the glucose ingested through constant monitoring. This operation may be complex especially for those who need to consume their meals in a restricted lapse of time, such as happens in many work environments. In addition, in order to obtain metabolic control, meals must be regular, as well as the measurements of the glucose levels and insulin administration. These requirements are difficult to reconcile with the habits of a young person and can generate concrete difficulties and discomfort in social interactions.

The need to control aspects of life which were previously considered "normal" can be experienced as a loss of freedom and spontaneity [37]. This is what some authors have called the frustration of chronicity [38], which makes diabetes a disease which can be managed but never defeated, and which has an impact on mood, as evidenced by the higher percentage of anxiety, depression and eating disorders among diabetic subjects. In particular, when the onset is compounded with other changes and transitions - such as adolescence [39] or aging - physical and social identity are affected. Some people may develop an image of their own body as suffering and "broken". Their body is different from the past and if compared to that of their peers, this diversity is interpreted in a negative way. Moreover, the external references impact on self-esteem and fear of judgment or contempt may force the patient to hide the symptoms of the disease from others as if they were something to be ashamed of.

Concerning behavior, one can observe different reactions, depending not only on the severity of the clinical situation, but also on personality, self-efficacy [40] and the social support they have [41]. For fear of being a burden, some patients isolate themselves, while others show provocative and hostile attitudes towards family and healthcare staff. One of the greatest risks, however, consists in the denial of the disease [42] and of the limitations that it entails. In order to maintain self-esteem, the patient avoids dealing with reality, calming, in his fragility, that he is omnipotent and refusing treatment. There is a risk that a vicious circle of poor compliance and metabolic decompensation will set in.

Early identification and treatment of these issues may help the patient develop an adaptive style for coping, which will give positive results on compliance and metabolic balance. In addition, it may prevent the risk of long term complications, which would further deteriorate the quality of life, and introduce new stressors and new blows to personal identity $[43,44]$.

\section{The Impact of Stress on the Disease Progression}

The role of stress in the etiology of diabetes is difficult to define and measure, but there is significant evidence of its metabolic consequences in individuals already suffering from chronic diseases, such as DM [45]. Psychological strain, in fact, activates neuroendocrine processes which influence the blood glucose level through the release 
of cortisol, growth hormone and endorphins. Faced with an external threat, blood glucose level increases, in order to mobilize energy. This reaction has an adaptive importance for a healthy organism, but, in diabetic patients, the stress-induced hyperglycemia may aggravate the disease since the hypoglycemic agents cannot counterbalance it.

These mechanisms mostly affect young people, in whom the endocrine system undergoes continuous adaptation, making them particularly sensitive to the effects of environmental stimuli [46]. On the other hand, in the case of the elderly, stress plays a major role in the development of complications such as neuropathy, nephropathy and retinopathy [47]. In addition, negative emotions may reduce and undermine the willingness to comply to treatment and to diets. Thus, a vicious circle of nervousness, poor compliance, poor glycemic control and physical vulnerability is established. This, in turn, makes it more difficult for people to cope with any new problems that arise $[48,49]$. On the contrary, early intervention on the acceptance of the disease seems to start a cycle of good compliance, adequate monitoring and further improvement of the patients' attitude towards diabetes. Therefore, care must be given through a process which supports resilience and personal self-efficacy, favoring the activation of problem-focused coping [50].

Treatment plans should also involve the families so they can help their family member to adapt to the illness. For instance, some research in developmental psychology has shown that there is a connection between parents' stress and that of their diabetic children [51] and this may influence the quality of glycemic control [52]. Family support is also important in adult patients: a partner's attitude, in fact, influences adherence to treatment [53] and the psychological impact of the disease has been shown to be more serious in type 2 diabetics who live alone [54].

\section{Therapeutic Approaches}

In view of the foregoing, we suggest that an effective cure for diabetes requires a global care plan which also takes into consideration the physical, relational and emotional aspects. Clinicians and patients must establish a collaborative relationship, requiring the clinicians to abandon any paternalism [55]. The main objective is to support the individuals' resources, making them protagonists in their own decisions and capable of making change [56,57]. Patients must feel they have equal dignity in the care administration process and that they are free to speak openly also about doubts and fears.

Taking charge of people with chronic disease is clinically challenging. It is therefore important that the working group be made up of different professionals with various responsibilities, including psychologists. They promote the acceptance of the disease, supporting the subjects in the management of conflicts and ambivalences as well as in the search of resources to promote resilience. Psychological support allows individuals to express emotions such as fear of the future, sadness for changes in lifestyle and anger [58]. Patients wonder why this misfortune has happened to them and may consider the disorder as an enemy to contend with, which cannot be defeated.

Special attention must be paid when the disease occurs in childhood and adolescence. The diagnosis of diabetes is critical both for the child and the parents. The family must be helped to understand the illness and to be aware of the importance of the treatment in everyday life. They must be helped to accept the implications of the situation, without however interfering with normal development milestones. Frequently, at the onset of the disease, children and caregivers express anxiety and depression, which may lead them to become too apprehensive with regard to care. Parents, in these cases, should be encouraged to promote - age permitting - the independence of their offspring, overseeing their social adaptation and self-esteem. There must be both collaboration and empowerment with teenagers, who need assistance in achieving autonomy in managing their therapy [59]. In most cases, they welcome the involvement of adults who give them support rather than oppress them. Hence, adults should reinforce positive behaviors and avoid excessive reproaches, never replacing their child in his decisions. Dealing with this age group, it is essential to always consider the patients' point of view, respecting their doubts, which often arise from the disease compounding with the adolescents' changes in body, feelings and thoughts. Gradually, the acceptance of the diagnosis allows patients to transform diabetes from an invincible enemy into a part of themselves with which they are able to make compromises. This new perspective generates a positive impact on emotions, behaviors and the overall outcome. The disease may remain a source of suffering and a personal limitation, but it can, nonetheless, still allow patients to take actions, build relationships and develop projects for the future.

\section{Conclusions}

This review examines recent literature about the psychological consequences of DM1 and DM2, with particular regard to the role played by stress and emotions. According to this literature, stress is often observed in conjunction with the diagnosis of diabetes and it alters the glucose metabolism and the immune response. Furthermore, the disease itself is a source of stress, because it requires considerable changes in lifestyle, thereby influencing the patient's identity. The ability to cope with these challenges, finally, may affect the actual management of the therapy and glycemic control. Our conclusion is that patients with DM require global care from a multidisciplinary team who is there to listen to their experiences and emotions, with the goal of helping them to accept and manage the disease.

\section{References}

1. Alberti KG, Zimmet PZ (1998) Definition, diagnosis and classification of diabetes mellitus and its complications. Part 1: diagnosis and classification of diabetes mellitus provisional report of a WHO consultation. Diabet Med 15: 539-553.

2. Holt TA, Kumar S (2010) ABC of diabetes. Oxford: Wiley-Blackwell.

3. Van Belle TL, Coppieters KT, Von Herrath MG (2011) Type 1 diabetes: etiology, immunology, and therapeutic strategies. Physiol Rev 91: 79-118.

4. Atkinson MA, Bluestone JA, Eisenbarth GS, Hebrok M, Herold KC, et al. (2011) How does type 1 diabetes develop?: the notion of homicide or $\beta$-cell suicide revisited. Diabetes 60: 1370-1379.

5. Hathout EH, Hartwick N, Fagoaga OR, Colacino AR, Sharkey J, et al. (2003) Clinical, autoimmune, and HLA characteristics of children diagnosed with type 1 diabetes before 5 years of age. Pediatrics 111: 860-863.

6. Porte D, Sherwin RS (1997) II diabete mellito. Guidotti, Pisa.

7. Wild S, Roglic G, Green A, Sicree R, King H (2004) Global prevalence of diabetes: estimates for the year 2000 and projections for 2030. Diabetes Care 27: 1047-1053.

8. Groop L (2000) Pathogenesis of type 2 diabetes: The relative contribution of insulin resistance and impaired insulin secretion. Int J Clin Pract Suppl 3-13. 
9. Selye $H$ (1985) The nature of stress. Basal Facts 7 : 3-11.

10. Zani B, Cicognani E (2000) Psicologia della salute. II Mulino, Bologna.

11. Pancheri P (1993) Stress, emozioni e malattia: introduzione alla medicina psicosomatica. Mondadori, Milano.

12. Fisher GN (2006) Trattato di psicologia della salute. Borla, Roma.

13. Nader N, Chrousos GP, Kino T (2010) Interactions of the Circadian CLOCK System and the HPA Axis. Trends Endocrinol Metab 21: 277-286.

14. Kajantie E, Philips DI (2006) The effects of sex and hormona status on the physiological response to acute psychosocial stress. Psychoneuroendocrinology 31: 151-178.

15. Traustadottir T, Bosch PR, Matt KS (2003) Gender differences in cardiovascular and hypothalamic-pituitary-adrenal axis responses to psychological stress in healthy older adult men and women. Stress 6: 133140.

16. Oktem O, Guzel Y, Aksoy S, Aydin E, Urman B (2015) Ovarian function and reproductive outcomes of female patients with systemic lupus erythematosus and the strategies to preserve their fertility. Obstet Gynecol Surv 70: 196-210.

17. Melmed S, Polonsky KS, Larsen PR, Kronenberg HM (2011) Williams Textbook of Endocrinology.

18. De Kloet ER, Joels M, Holsboer F (2005) Stress and the brain: from adaptation to disease. Nat Rev Neurosci 6: 463-475.

19. Elenkov IJ, Chrousos GP (1999) Stress Hormones, Th1/Th2 patterns, Pro/ Anti-inflammatory Cytokines and Susceptibility to Disease. Trends Endocrino Metab 10: 359-368

20. Atanackovic D, Nowottne U, Freier E, Weber CS, Meyer S, et al. (2013) Acute psychological stress increases peripheral blood CD3 + CD56 + natural killer T cells in healthy men: possible implications for the development and treatment of allergic and autoimmune disorders. Stress 16: 421-428.

21. Elenkov IJ, Chrousos GP (2002) Stress hormones, proinflammatory and antiinflammatory cytokines, and autoimmunity. Ann N Y Acad Sci 966: 290 303.

22. Dube SR, Fairweather D, Pearson WS, Felitti VJ, Anda RF, et al. (2009) Cumulative childhood stress and autoimmune diseases in adults. Psychosom Med 71: 243-250.

23. Carlsson E, Frostell A, Ludvigsson J, Faresjö M (2014) Psychological stress in children may alter the immune response. J Immunol 192: 2071-2081.

24. Miller GE, Chen E, Parker KJ (2011) Psychological stress in childhood and susceptibility to the chronic diseases of aging: moving toward a model of behavioral and biological mechanisms. Psychol Bull 137: 959-997.

25. Nygren M, Ludvigsson J, Carstensen J, Sepa Frostell AS (2013) Family psychological stress early in life and development of type 1 diabetes: The ABIS prospective study. Diabetes Res Clin Pract 100: 257-264.

26. Kassi E, Pervanidou P, Kaltsas G, Chrousos G (2011) Metabolic syndrome: definitions and controversies. BMC Med 9:48.

27. Kaur J (2014) A comprehensive review on metabolic syndrome. Cardiol Res Pract 2014: 943162

28. Expert Panel on Detection, Evaluation and Treatment of High Blood Cholesterol in Adults (2001) Executive summary of the third report of the national cholesterol education program (NCEP) Expert Panel on Detection, Evaluation, And Treatment of High Blood Cholesterol In Adults (Adult Treatment Panel III). JAMA 285: 2486-2497.

29. Alberti KG, Zimmet $P$, Shaw J (2005) IDF epidemiology task force consensus group. The metabolic syndrome--a new worldwide definition. Lancet 366 1059-1062.

30. Alberti KG, Eckel RH, Grundy SM, Zimmet PZ, Cleeman JI, et al. (2009) Harmonizing the metabolic syndrome. A joint interim statement of the International Diabetes Federation Task Force on Epidemiology and Prevention; National Heart, Lung, And Blood Institute; American Heart Association; World Heart Federation; International Atherosclerosis Society and International Association for the Study of Obesity. Circulation 120: 16401645.

31. Li J, Jarczok MN, Loerbroks A, Schöllgen I, Siegrist J, et al. (2013) Work stress is associated with diabetes and prediabetes: cross-sectional results from the MIPH Industrial Cohort Studies. Int J Behav Med 20: 495-503.

32. Heraclides AM, Chandola T, Witte DR, Brunner EJ (2012) Work stress, obesity and the risk of Type 2 Diabetes: gender-specific bidirectional effect in the Whitehall II Study. Obesity (Silver Spring) 20: 428-433.

33. Heraclides A, Chandola T, Witte DR, Brunner EJ (2009) Psychosocial stress at work doubles the risk of Type 2 Diabetes in middle-aged women. Diabetes Care 32: 2230-2235.

34. Aschbacher K, Kornfeld S, Picard M, Puterman E, Havel PJ, et al. (2014) Chronic stress increases vulnerability to diet-related abdominal fat, oxidative stress, and metabolic risk. Psychoneuroendocrinology 46: 14-22.

35. Beardsley G, Goldstein MG (1993) Psychological factors affecting physica condition. Endocrine disease literature review. Psychosomatics 34: 12-19.

36. Bergmann N, Gyntelberg F, Faber J (2014) The appraisal of chronic stress and the development of the metabolic syndrome: a systematic review of prospective cohort studies. Endocr Connect 3: R55-R80.

37. Pearce MJ, Pereira K, Davis E (2013) The psychological impact of diabetes: A practical guide for the nurse practitioner. J Am Assoc Nurse Pract 25: 578583.

38. Stankovic Z, Jašovic-Gašic M, Lecic-Toševski D (2013) Psychologica problems in patients with type 2 diabetes - Clinical considerations. Vojnosanit Pregl 70: 1138-1144.

39. Tran V, Wiebe DJ, Fortenberry KT, Butler JM, Berg CA (2011) Benefit finding affective reactions to diabetes stress, and diabetes management among early adolescents. Health Psychol 30: 212-219.

40. Wardian J, Sun F (2014) Factors associated with diabetes-related distress: implications for diabetes self-management. Soc Work Health Care 53: 364381.

41. Karlsen B, Bru E (2014) The relationship between diabetes-related distress and clinical variables and perceived support among adults with type 2 diabetes: A prospective study. Int J Nurs Stud 51: 438-447.

42. Garay-Sevilla ME, Malacara JM, Gutierrez-Roa A, Gonzalez E (1999) Denial of disease in Type 2 diabetes mellitus: its influence on metabolic control and associated factors. Diabet Med 16: 238-244.

43. Fejfarová V, Jirkovská A, Dragomirecká E, Game F, Bém R, et al. (2014) Does the diabetic foot have a significant impact on selected psychological or social characteristics of patients with diabetes mellitus? J Diabetes Res 2014: 371938.

44. Maiorino MI, Bellastella G, Esposito K (2014) Diabetes and sexua dysfunction: current perspectives. Diabetes Metab Syndr Obes 7: 95-105.

45. Piłaciński S, Zozulińska-Ziółkiewicz DA (2014) Influence of lifestyle on the course of type 1 diabetes mellitus. Arch Med Sci 10: 124-134.

46. Berlin KS, Rabideau EM, Hains AA (2012) Empirically derived patterns of perceived stress among youth with Type 1 Diabetes and relationships to metabolic control. J Pediatr Psychol 37: 990-998.

47. Surwit RS, Schneider MS (1993) Role of stress in the etiology and treatment of diabetes mellitus. Psychosom Med 55: 380-393.

48. Pandit AU, Bailey SC, Curtis LM, Seligman HK, Davis TC, et al. (2014) Disease-related distress, self-care and clinical outcomes among low-income patients with diabetes. J Epidemiol Community Health 68: 557-564.

49. Shah BM, Gupchup GV, Borrego ME, Raisch DW, Knapp KK (2012) Depressive symptoms in patients with Type 2 Diabetes Mellitus: do stress and coping matter? Stress Health 28: 111-122.

50. Anderson JB, Rubin RR (2004) Psicologia pratica per diabetologi. Tecniche comportamentali efficaci. II Poligrafo. 
51. Sweenie R, Mackey ER, Streisand R (2014) Parent-child relationships in Type 1 Diabetes: associations among child behavior, parenting behavior, and pediatric parenting stress. Fam Syst Health 32: 31-42.

52. Tsiouli E, Alexopoulos EC, Stefanaki C, Darviri C, Chrousos GP (2013) Effects of diabetes-related family stress on glycemic control in young patients with type 1 diabetes: Systematic review. Can Fam Physician 59: 143-149.

53. Pereira MG, Pedras S, Machado JC (2014) Family variables as moderators between beliefs towards medicines and adherence to self-care behaviors and medication in Type 2 Diabetes. Fam Syst Health 32: 198-206.

54. Hara Y, Hisatomi M, Ito H, Nakao M, Tsuboi K, et al. (2014) Effects of gender, age, family support, and treatment on perceived stress and coping of patients with type 2 diabetes mellitus. BioPsychoSoc Med 8: 16.
55. Gentili P, Di Berardino P, Parmentola C (2006) L'approccio psicologico nel diabete. Il dialogo, le teorie, l'esperienza. In Pagina, Milano.

56. Caracci G, Garzaniga S (2010) I risultati della ricerca AGENAS. Definizione, modello di analisi, strumenti di rilevazione ed esperienze significative di empowerment in sanità. I Quaderni di Monitor 25: 10-18.

57. Zucconi A, Howell (2003) La promozione della salute. Un approccio globale per il benessere della persona e della società. La meridiana, Bari.

58. Margiotta A, Manna RM, Biotti A (2007) Ruolo dello psicologo nei servizi di diabetologia. Giornale Italiano di Diabetologia e Metabolismo 27: 166-174.

59. Delamater AM (2009) Psychological care of children and adolescents with diabetes. Pediatr Diabetes 10 (Suppl 12): 175-184.

\section{Acknowledgements}

The Authors wish to thank Mrs Anna Racca for the linguistic editing contribution. 